\title{
Neuroprotective effects of the new Na channel blocker rs100642 in global ischemic brain injury
}

Suat Kamisli ${ }^{1}$, Cenk Basaran ${ }^{1}$, Kadir Batcioglu², Mustafa Namık Oztanir ${ }^{1}$, Mehmet Gul ${ }^{3}$, Basri Satilmis², Ayse Burcin Uyumlu², Basak Kayhan ${ }^{4}$, Metin Genc ${ }^{5}$

${ }^{1}$ Division of Neurology, Faculty of Medicine, İnönü University, Malatya, Turkey ${ }^{2}$ Division of Biochemistry, Faculty of Medicine, İnönü University, Malatya, Turkey ${ }^{3}$ Division of Histology, Faculty of Medicine, İnönü University, Malatya, Turkey ${ }^{4}$ Division of Medicinal Biology and Genetics, Faculty of Medicine, Inönü University, Malatya, Turkey

${ }^{5}$ Division of Public Health, Faculty of Medicine, Inönü University, Malatya, Turkey

Submitted: 21 September 2016

Accepted: 18 April 2017

Arch Med Sci 2019; 15, 2: 467-474

DOI: https://doi.org/10.5114/aoms.2017.72550

Copyright $\odot 2019$ Termedia \& Banach

\section{Abstract}

Introduction: RS100642, a mexiletine analogue, is a novel sodium channel blocker with neuroprotective and antioxidant activities. The protectivity of RS100642, which has been shown against focal cerebral ischemia, was investigated in global cerebral ischemia in this study.

Material and methods: Global cerebral ischemia was induced for five minutes in adult male Wistar Albino rats via the 4-vessel occlusion method. Intravenous administration of $1 \mathrm{mg} / \mathrm{kg}$ RS100642 following reperfusion for 30 min (RS100642 group) was compared with a sham treatment group (ischemia group) and nonischemized group (control) histologically based on morphology and caspase- 3 immunohistochemistry, and biochemically based both on measurement of oxidative stress including malondialdehyde (MDA) levels, superoxide dismutase (SOD), glutathione peroxidase (GPx) and catalase (CAT) activities and on assessment of apoptosis including caspase- 3 and -8 activities and tumor necrosis factor $\alpha$ (TNF- $\alpha$ ) levels at the end of $6 \mathrm{~h}$. Results: While the RS100642 group had significantly lower MDA levels and higher SOD activities than the sham treatment group $(p<0.05)$, GPx and CAT activities of the RS100642 and sham treatment groups were similar $(p>0.05)$ and significantly lower than those of the controls $(p<0.05)$. Necrosis and caspase- 3 activity and immunoreactivity in the RS100642 group were significantly lower than those in the sham treatment group $(p<0.05)$, while there was no significant difference between groups regarding caspase- 8 and TNF- $\alpha(p>0.05)$.

Conclusions: $\mathrm{Na}^{+}$channel blockade by RS100642 has remarkable neuroprotective effects following global brain ischemia/reperfusion damage. Further research is required to determine the optimum dose and time of administration.

Key words: brain injury, global cerebral ischemia, sodium channels, neuroprotection.

\section{Introduction}

Ischemic cerebrovascular events cause significant morbidity and mortality [1]. Ischemia results in energy failure followed by loss of cell membrane pump functions, disruption of ion homeostasis, intracellular

\author{
Corresponding author: \\ Suat Kamisli MD \\ Division of Neurology \\ Faculty of Medicine \\ İnönü University \\ 44280 Malatya, Turkey \\ Phone: \\ +90 422 3410660-4904 \\ E-mail: suat.kamisli@ \\ hotmail.com
}


accumulation of $\mathrm{Na}^{+}$and $\mathrm{Ca}^{2+}$, acidosis, glutamate excitotoxicity, free radical damage, accumulation of arachidonate oxidation products, glial activation, breakdown of the blood-brain barrier, leukocyte infiltration, and activation of caspases [2-4]. Cell death may occur through both apoptosis and necrosis $[5,6]$.

The only current clinical measure of preventing persistent brain damage following ischemia is immediate reperfusion, comprising resuscitation within seconds to minutes in global ischemia and thrombolysis within minutes to a few hours in focal ischemia. Hypothermia is also widely used as an adjunctive measure of treatment. However, both are limited by the difficulty of administration, shortness of time, inefficiency, and unwanted side effects. Furthermore, reperfusion is not singlehandedly life-saving, and even the reverse may be true when the severity of brain damage increases, leading to the development of ischemia/reperfusion (I/R) damage [7]. This situation has led to the search for neuroprotective drugs. Unfortunately, among more than 50 different drugs evaluated for neuroprotection, none yet has been proven to be sufficient for clinical use $[4,8]$.

RS100642 (1-(2',6'-dimethyl-phenoxy)-2-ethylaminopropane hydrochloride) is a mexiletine analogue with less cardiotoxicity and neurotoxicity than those observed with mexiletine [9]. It blocks sodium channels with binding properties similar to mexiletine $\left(\mathrm{p} K_{\mathrm{i}}=5.09 \mu \mathrm{M}\right)$ but exhibits a more potent use-dependent reduction in compound action potentials as determined by rat vagus nerve preparations [10]. RS100642 has been examined in in vivo models of focal cerebral ischemia and was found to be beneficial in terms of neuroprotection not only in reducing the infarct size but also regulating the expression of $\mathrm{Na}_{v} 1.1$ channel protein, reflecting that the effect results directly from $\mathrm{Na}^{+}$channel blockade $[11,12]$. However RS100642 has not yet been tried clinically, nor has it been studied in in vivo global brain ischemia experimentally.

Therefore, this study aimed to evaluate the potential neuroprotective effects of RS100642 in global transient ischemia.

\section{Material and methods}

\section{Animals and surgical procedure}

Twenty-seven male Wistar Albino rats aged 7-9 months and weighing 229-308 g were obtained from the Experimental Animal Breeding and Research Center of Inonu University. Prior to the study, all animals were acclimatized to the housing conditions for 1 week on a 12-hour light/ dark cycle at $18-22^{\circ} \mathrm{C}$ room temperature in cages sized $40 \times 21 \times 15 \mathrm{~cm}$ with 5 rats in each cage and fed with ad libitum laboratory chow and tap water. The experimental protocol was approved by the Ethics Committee for Animal Experiments of Inonu University. The rats were divided into 3 groups, namely control $(n=7)$, ischemia $(n=10)$ and RS100642 $(n=10)$. RS100642 was obtained from Roche Bioscience (Palo Alto, CA, USA). The compound was dissolved in a vehicle of distilled, deionized water immediately prior to testing.

Global ischemia was performed according to the 4-vessel occlusion method defined by Pulsinelli and Brierley [13]. Briefly, on the $1^{\text {st }}$ day, all rats were anesthetized with ketamine + xylazine intraperitoneal (i.p.), followed by midsagittal incisions on the backs of their necks behind the occipital bones, dissection of the paravertebral muscles, and electrocauterization of both vertebral arteries. After electrocauterization, the rats were placed on their backs, and ventral cervical midsagittal incisions were made, followed by placement of loose silk sutures around both carotids, and suturing of the incisions. On the $2^{\text {nd }}$ day, the rats were briefly anesthetized with ether. In the controls, only the sutures were removed and incisions were re-sutured, inducing no global brain ischemia. On the other hand, the rats in both ischemia and RS100642 groups had their sutures removed, both their carotids concomitantly were clasped tightly for $5 \mathrm{~min}$, and the clasps were then removed for reperfusion. After $30 \mathrm{~min}$ of reperfusion, the RS100642 group rats received $1 \mathrm{mg} / \mathrm{kg}$ intravenous (i.v.) RS100642 at a volume of $1 \mathrm{ml}$ $\mathrm{kg}$, while the ischemia group rats received i.v. isotonic saline at the same volume. The rats in both ischemia and RS100642 groups then had their incisions re-sutured and were left to recover. No specific premedication other than anesthesia was given prior to surgery, and paracetamol $300 \mathrm{mg} / \mathrm{kg}$ i.p. was administered postsurgically for analgesia. Three out of ten rats in the ischemia group and one out of seven rats in the control group were excluded from the study due to peri-operative mortality. Therefore, the investigations were conducted in 6 rats in the controls, 7 rats in the ischemia group, and 10 rats in the RS100642 group.

At the $6^{\text {th }}$ hour following the procedure, all rats were sacrificed via decapitation under deep ether anesthesia. The brains were removed, the left hemispheres being preserved for $24 \mathrm{~h}$ at $-40^{\circ} \mathrm{C}$ for biochemical investigations, and the right hemispheres being preserved for $24 \mathrm{~h}$ in $10 \%$ formalin for histopathological examinations.

\section{Biochemical measurements}

The left hemispheres of the brain samples were promptly dissected and perfused with $50 \mathrm{mM}(\mathrm{pH}$ : 7.4) cold phosphate buffer saline solution (PBS). Samples were homogenized in $1 / 5(\mathrm{w} / \mathrm{v})$ PBS. The 
homogenate was sonicated three times for $30 \mathrm{~s}$ with intervals and centrifuged at $20,000 \times \mathrm{g}$ for 15 min. Supernatants were separated and kept at $-80^{\circ} \mathrm{C}$ until other biochemical measurements were performed.

The protein concentrations were determined according to the method of Lowry et al. [14], the malondialdehyde (MDA) levels were determined according to the method of Mihara and Uchiyama [15], the superoxide dismutase (SOD) activity was determined using the method of McCord and Fridovich [16], the catalase (CAT) activity was determined with the method of Luck [17], and the glutathione peroxidase (GPx) activity was measured with the method of Lawrence and Burk [18].

The activities of caspases- 3 and -8 and tumor necrosis factor (TNF)- $\alpha$ levels were measured using ELISA kits (BioVision Research Product, CA, USA and eBioscience Research Kits, CA, USA, respectively) according to the manufacturer's instructions.

\section{Histopathological evaluations}

The brain samples were placed in $10 \%$ buffered formalin and embedded in paraffin. Sections of brain tissues were cut at $6 \mu \mathrm{m}$ and mounted on slides and stained with hematoxylin-eosin $(\mathrm{H}+\mathrm{E})$ and immunohistochemically with the Rabbit Polyclonal Antibody to Active Caspase-3 (Ab4051) Kit. The Caspase-3 Kit was used as recommended by the manufacturer (Abcam, Cambridge, United Kingdom). All sections were systematically examined with a Leica DFC 280 light microscope and analyzed in the Leica Q Win Plus V3 Image Analysis System (Leica Micros Imaging Solutions Ltd.; Cambridge, UK).

We applied modified criteria developed by Grafe et al. and Radovsky et al., who outlined light microscopic structural features of neurons and brain tissues $[19,20]$. By light microscopy, necrotic (ischemic) neurons can be identified as exhibiting nuclear pyknosis and cytoplasmic eosinophilia. Other cellular alterations such as hyperchromatic indented or sharply scalloped nucleus and swollen neurons were also included in the neuronal counts.

Histopathological injury (mononuclear, polymorphonuclear, macrophages infiltrate and hemorrhage and edema (cells swell up) and gliosis) was scored in brain sections according to the following semi-quantitative scale: normal $=0$, mild injury = 1 (infiltration, hemorrhage, edema and gliosis area $<10 \%$ of total brain cortex area), moderate injury $=2$ (infiltration, hemorrhage, edema and gliosis area $11 \%$ to $20 \%$ of total brain cortex area), severe injury $=3$ (infiltration, hemorrhage, edema and gliosis area $21 \%$ to $40 \%$ of total brain cortex area); and complete cavitary infarct
$=4$ (infiltration, hemorrhage, edema and gliosis area $>41 \%$ of total brain cortex area). Necrotic (ischemic) neurons in affected neuronal populations were counted without regard for the total number of neurons in the region, and put into five ranked categories as follows: 0 necrotic neurons $=0 ; 1-15=(+1) ; 16-30=(+2) ; 31-50=(+3)$; and $>50=(+4)$ randomly at 20 different areas in the brain cortex with the $20 \times$ objective.

The $\mathrm{H}$-Score method was used to score the degree of caspase-3 positive immunoreactivity of neurons and glial cells in total sections' area of the brain cortex. This immunohistochemical semi-quantitative method consists of the percentages of positively stained cells multiplied by a weighted intensity of staining: $\mathrm{H} \mathrm{Score}=P i(i+1)$, where $P i$ is the percentage of stained cells in each intensity category (0-100\%), and $i$ is the intensity indicating weak $(i=1)$, moderate $(i=2)$ or strong staining $(i=3)[21]$.

\section{Statistical analysis}

Statistical analysis was performed using SPSS for Windows 15.0 software (SPSS Inc., IL, USA). For analysis of biochemical parameters, comparison among groups was made with the analysis of variance (ANOVA) test, while post hoc intergroup comparisons were performed with the Tukey HSD test. $P<0.05$ was regarded as statistically significant.

\section{Results}

\section{Evaluation of biochemical parameters}

\section{Oxidative stress}

The definitive statistical data regarding biochemical parameters are displayed in Table I. Post hoc comparison of the groups against each other with the Tukey HSD test revealed a significant decrease in the SOD activities in the ischemia group as compared with both the RS100642 group and the controls $(p<0.05)$. On the other hand, SOD activities did not differ significantly between the RS100642 and the control groups ( $p>0.05)$. Also, MDA level in the RS100642 group differed significantly compared to that of the ischemia group. Contrastingly, the CAT and GPx activities were significantly lower in both ischemia and RS100642 groups as compared with the controls ( $p<0.05)$, while the RS100642 group was not significantly different from the ischemia group with regard to both of these parameters $(p>0.05)$.

\section{Apoptosis}

Caspase- 3 and -8 activities and TNF- $\alpha$ levels are displayed in Table II. Post hoc comparison of the groups against each other with the Tukey 
Table I. Results are displayed as mean \pm SD. There was significantly lower SOD activity in the ischemia group compared to the control group and a significantly higher value in the RS100642 group compared to the ischemia group. Also, a significantly lower MDA level was found in the RS100642 group compared to the ischemia group. On the other hand, the GPx and CAT activities of both RS100642 and ischemia groups were significantly lower than those of the controls

\begin{tabular}{|lcccc|}
\hline Groups $(n)$ & $\begin{array}{c}\text { CAT } \\
\text { [U/mg protein] }\end{array}$ & $\begin{array}{c}\text { SOD } \\
\text { [U/mg protein] }\end{array}$ & $\begin{array}{c}\text { GPx } \\
{[\text { U/mg protein] }}\end{array}$ & $\begin{array}{c}\text { MDA } \\
\text { [nmol/mg protein] }\end{array}$ \\
\hline Control (6) & $636.15 \pm 133.20$ & $5.52 \pm 0.47$ & $1.65 \pm 0.33$ & $20.79 \pm 2.35$ \\
\hline Ischemia (7) & $473.31 \pm 138.02^{*}$ & $4.74 \pm 0.22^{*}$ & $1.04 \pm 0.14^{*}$ & $24.84 \pm 1.98$ \\
\hline RS100642 (10) & $457.99 \pm 57.85^{*}$ & $5.36 \pm 0.55^{\#}$ & $1.05 \pm 0.15^{*}$ & $19.35 \pm 4.25^{\#}$ \\
\hline
\end{tabular}

${ }^{*} p<0.05$ compared with control; ${ }^{*} p<0.05$ compared with ischemia.

Table II. Results are displayed as mean \pm SD. There was significantly higher caspase-3 activity in the ischemia group as compared with the RS100642 and control groups, while the caspase-3 activities of RS100642 and control groups were similar. Caspase- 8 activities and TNF- $\alpha$ levels did not differ significantly between groups

\begin{tabular}{|lccc|}
\hline Groups $(n)$ & $\begin{array}{c}\text { Caspase-3 } \\
\text { [RFU/mg protein] }\end{array}$ & $\begin{array}{c}\text { Caspase-8 } \\
\text { [RFU/mg protein] }\end{array}$ & $\begin{array}{c}\text { TNF- } \alpha \\
\text { [pg/ml] }\end{array}$ \\
\hline Control (6) & $14.79 \pm 1.51$ & $53.04 \pm 13.80$ & $4.86 \pm 1.07$ \\
\hline Ischemia (7) & $19.91 \pm 2.43^{*}$ & $65.74 \pm 19.18$ & $5.62 \pm 1.49$ \\
\hline RS100642 (10) & $16.84 \pm 2.83^{\#}$ & $65.21 \pm 9.24$ & $4.35 \pm 1.32$ \\
\hline
\end{tabular}

${ }^{*} p<0.05$ compared with control; ${ }^{*} p<0.05$ compared with ischemia.

HSD test revealed significantly higher caspase-3 activity in the ischemia group as compared with the RS100642 and control groups ( $p<0.05)$. On the other hand, no significant difference was found among the ischemia, control and RS100642 groups with regard to caspase- 8 and TNF- $\alpha$ levels $(p>0.05)$.

\section{Evaluation of histopathological findings}

The samples of the control group were normal in histological appearance (Figures $1 \mathrm{~A}, \mathrm{~B}$ ). The ratio of ischemic to healthy neurons, neuronal swelling, tissue edema, and other signs of histopathological injury including hemorrhage and infiltration (Figure 2 A) and caspase-3 positive immunoreactivity (Figure $2 \mathrm{~B}$ ) in the ischemia group were significantly higher than in the control and RS100642 groups. The number of ischemic neu-

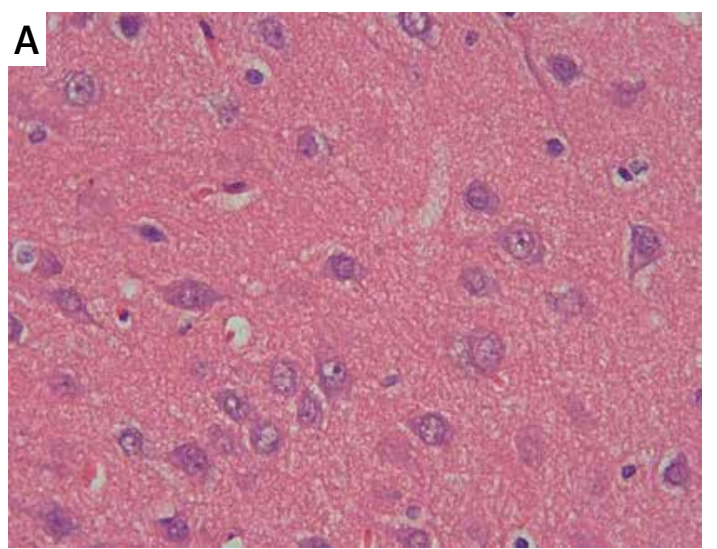

rons, the severity of the histopathological injury and caspase- 3 positive immunoreactivity were significantly lower in the RS100642 group (Figures $3 \mathrm{~A}, \mathrm{~B})$ as compared with the ischemia group. The total score and $\mathrm{H}$-score values in each group are shown in Figure 4.

\section{Discussion}

We have demonstrated a promising neuroprotective effect of RS100642 against I/R injury in global ischemia of 5 min followed by reperfusion for $30 \mathrm{~min}$, reflected strongly in the SOD activities, and partially in caspase- 3 levels and histopathology. To date, RS100642 has been investigated in focal ischemia scarcely, and this is the first study of this agent in global ischemia. Global cerebral ischemia with 4-vessel blockade has been induced in several studies via clamping the carotids from



Figure 1. Control group: A - the controls with the normal histological appearance of brain tissue. $H+E, 40 \times$, B - absence of caspase-3 positive immunoreactivity, caspase-3, 40x 

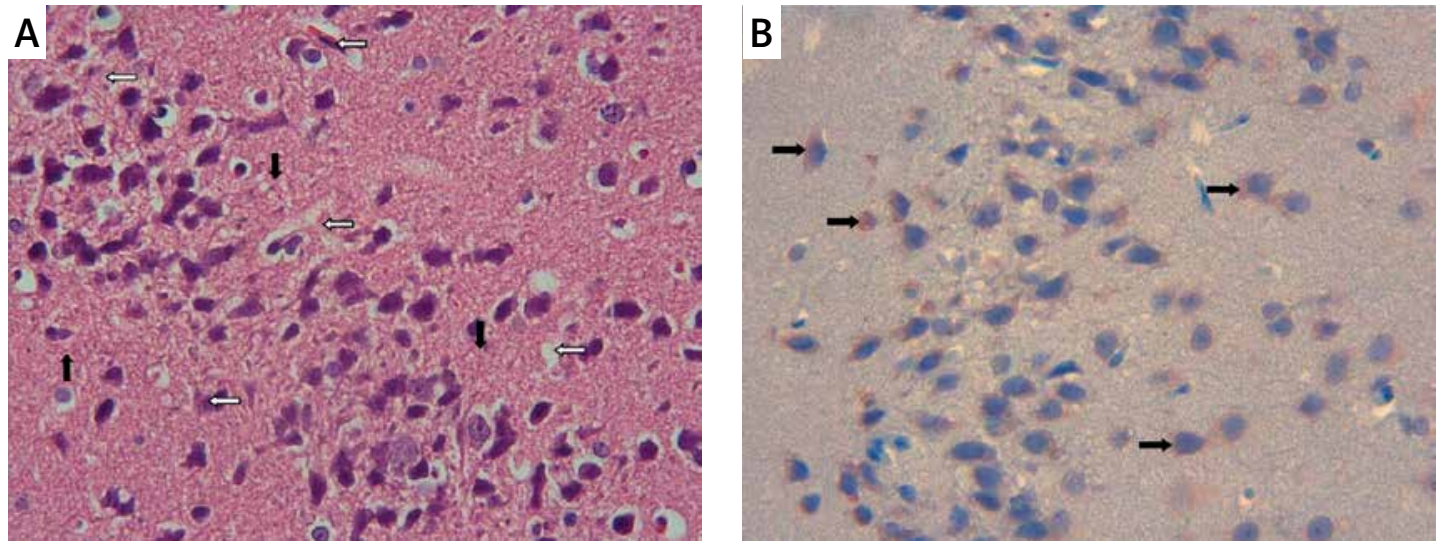

Figure 2. Ischemia group: A - necrotic (white arrows) and swollen neurons (black arrows). $H+E, 40 x, B$ - prominent caspase-3 positive immunoreactivity (black arrows). Caspase-3, 40x
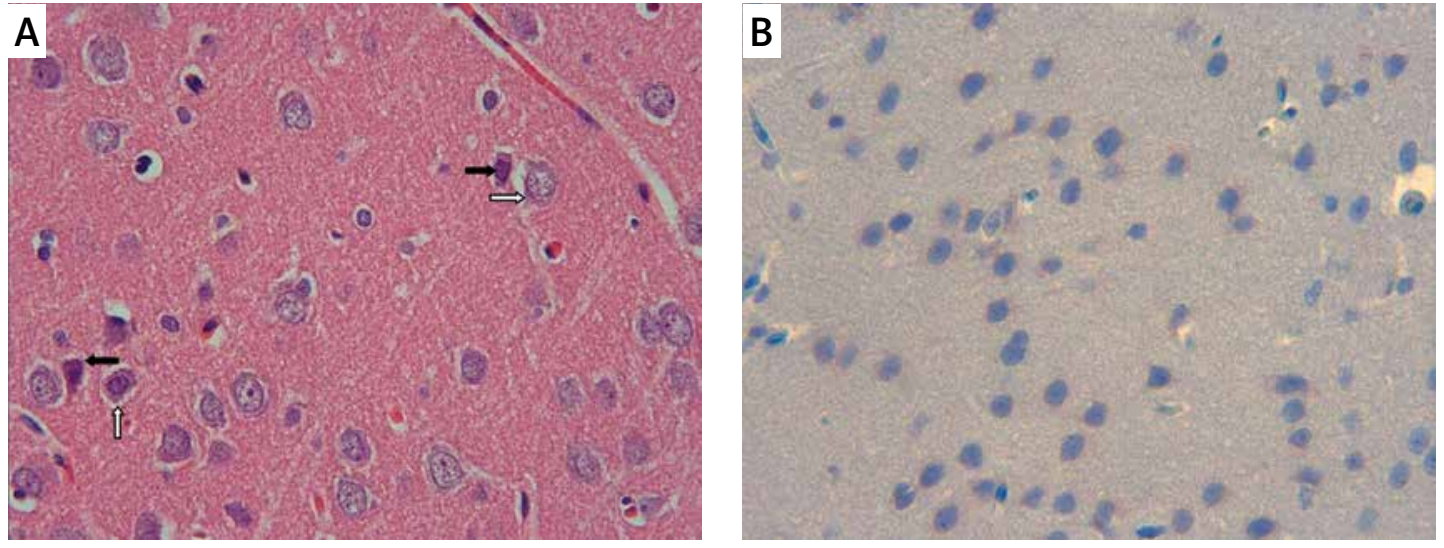

Figure 3. RS100642 group: A - necrotic neurons (white arrows), with minimal perineural edema (white arrows). $\mathrm{H}+\mathrm{E}, 40 \times$, B - slight caspase-3 positive immunoreactivity. Caspase-3, 40x

10 to $30 \mathrm{~min}[22,23]$. Cell death has been shown to take place in minutes after the initiation of global ischemia, and this is approximately $5 \mathrm{~min}$ in most sensitive parts of the central nervous system [24]. Therefore, we clasped both carotids concomitantly for $5 \mathrm{~min}$ to produce brain injury. We sacrificed the rats at the $6^{\text {th }} \mathrm{h}$, as this duration is adequate for superoxide radical accumulation [25], disruption of the cell membrane, and disintegration of organelles in I/R damage [26]. We administered RS100642 at a dose of $1 \mathrm{mg} / \mathrm{kg}$, the same dose that was found to be successfully neuroprotective in previous studies. Lower doses have also been tested in focal ischemia and were found to be less effective, while increasing the dose further was not beneficial [9].

Under physiological conditions, the brain is an organ with high $\mathrm{O}_{2}$ consumption and free oxygen radical production, but low antioxidant capacity. Due to the abundance of polyunsaturated fatty acids, it is particularly damaged by oxidative stress during I/R injury. Increased production of reactive oxygen species (ROS) due to I/R injury may easily overcome the antioxidant systems and result in oxidative damage to cellular components [25]. In-

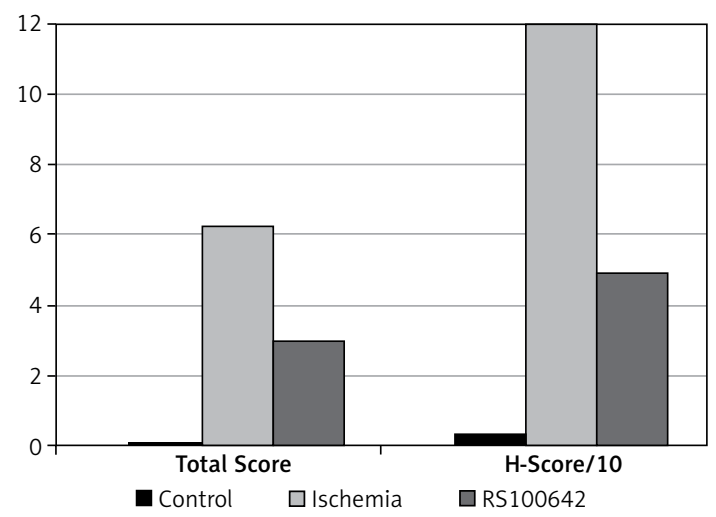

Figure 4. Comparative graphics of brain tissue histopathological injury as total and $\mathrm{H}$-score in each experimental group (mean $\pm \mathrm{SD}$ )

creases in MDA levels and decreases in antioxidant enzyme activities in traumatic brain damage have been reported previously [27]. Therefore the MDA levels and SOD, CAT and GPx activities are reliable indicators of cerebral I/R damage. In our study, RS100642 suppressed early accumulation of MDA and enhanced the activity of SOD. The decrease in MDA levels reflects decreased lipid peroxidation, whereas the lower level of SOD activity reflects 
reduction in dismutation of superoxide radicals. However, RS100642 failed to prevent reduction of CAT and GPx levels significantly. Like SOD activity being used up against increasing $\mathrm{O}_{2}^{-}$levels, CAT and GPx activities are used up in order to reduce the increasing $\mathrm{H}_{2} \mathrm{O}_{2}$ levels [28, 29]. Neither CAT nor GPx activities differed significantly from those in the ischemia group with RS100642 administration, indicating that administration of RS100642 significantly protects from damage related to lipid peroxidation and $\mathrm{O}_{2}^{-}$, but not $\mathrm{H}_{2} \mathrm{O}_{2}$, which is regarded as the main radical responsible for necrosis [30,31]. Necrosis occurs when intracellular ATP levels fall below $25 \%$ of the basal levels, and apoptosis commences if they remain higher [32]. Unless eliminated by $\mathrm{CAT}, \mathrm{H}_{2} \mathrm{O}_{2}$ leads to failure of ATP synthesis via overloading of the cellular hexose monophosphate pathway for reduction of GS-SG and depletion of nicotinamide adenine dinucleotide $\left(\mathrm{NAD}^{+}\right)$but also directly suppresses ATP synthesis in the mitochondria [30], facilitates iron-mediated secondary free $\mathrm{O}_{2}$ radical formation, and peroxidizes cell lipids [33]. As mitochondria play a pivotal role in both $\mathrm{Ca}^{2+}$ metabolism and initiation of caspase cascade, reduction in ATP synthesis and increase in mitochondrial permeability lead to increased cytochrome $c$ release and therefore apoptosis accompanied by poly (ADP-ribose) polymerase (PARP)- and c-Jun N-terminal kinase (JNK)-mediated necroptosis, a condition with features of both necrosis and apoptosis [2, 34, 35]. These effects of $\mathrm{H}_{2} \mathrm{O}_{2}$ may explain why necrosis persisted morphologically following RS100642 administration. The difference in parameters related to $\mathrm{H}_{2} \mathrm{O}_{2}$ and $\mathrm{O}_{2}^{-}$may arise from a free radical scavenging property of RS100642, which does not act on $\mathrm{H}_{2} \mathrm{O}_{2}$ and peroxynitrite. Although it has not yet been investigated whether RS100642 has free radical scavenging activity, mexiletine has been shown to be a potent free radical scavenger [36]. Further studies investigating whether RS100642 has free radical scavenging activity and whether the introduction of RS100642 in the company of a scavenger against $\mathrm{H}_{2} \mathrm{O}_{2}$ could be more beneficial are required to clarify these questions.

Caspase-dependent apoptosis may be initiated either by activation of caspase- 8 by death receptors on the cell surface or by activation of caspase- 3 through mitochondrial stress including cytochrome c (Cyt c) release [37]. Cyt c released from the mitochondria forms a complex with apoptosis activating factor- 1 and pro-caspase-9, resulting in caspase- 3 activation, cleavage of cellular substrates and cell death, as demonstrated in the delayed neuronal death following ischemia [38]. Translocation of Cyt $c$ and activation of caspase- 3 have been reported following global ischemia in gerbils [39], and following transient middle cerebral artery occlusion for $2 \mathrm{~h}$, starting as early as the $30^{\text {th }}$ min of reperfusion [40]. Accordingly, increased caspase- 3 immunoreactivity in the cerebral cortex after cerebral I/R injury has been demonstrated histopathologically [41]. Likewise, histopathological images support our biochemical findings. While the brain specimens from control animals presented no histological alterations (Figures $1 \mathrm{~A}, \mathrm{~B}$ ), those from animals in the ischemia group displayed necrotic and swollen neurons with partial edema as well as prominent caspase-3 positive immunoreactivity (Figures 2 A, B). The inhibition of histopathological changes observed in the RS100642 group is illustrated in Figures $3 \mathrm{~A}$ and $\mathrm{B}$. It has been demonstrated that neurons derived from caspase-3-knockout mice are resistant to ischemic stress [42], and caspase inhibitors (z-DEVD-FMK or Z-VAD-FMK) prevent ischemia-induced neuronal damage and improve neurological outcome [43]. Our results confirmed the increased caspase- 3 activity and immunoreactivity following I/R injury in rats and demonstrated a beneficial effect of RS100642. The second pathway of apoptosis involves activation of caspase- 8 through death receptors located on the cell surface. The death receptors belong to the tumor necrosis factor (TNF) superfamily, and the best-characterized ones are CD95 (or Fas), TNF receptor-1 and TNF-related apoptosis-inducing ligand (TRAIL) receptors DR4 and DR5. Cytotoxic effects of high levels of TNF- $\alpha$ are mediated either by its inflammatory action or binding to TNF receptor 1 (TNFR1)-type receptors on dopaminergic neurons leading to activation of caspase-8-mediated apoptosis [44]. TNF- $\alpha$ can also induce the activation of inducible nitric oxide synthase (iNOS), amplifying the inflammatory response and leading to the production of more cytokines [45]. We observed considerable but statistically insignificant increases in TNF- $\alpha$ and caspase- 8 levels in the ischemia group as compared with the controls, while these increases were particularly inhibited in the group with $\mathrm{Na}^{+}$channel blockade. We, therefore, propose that apoptosis develops following global brain ischemia mainly by caspase- 3 activation through the mitochondrial pathway rather than activation of death receptors, and can be prevented by RS100642 administration.

In conclusion, our results suggest that $\mathrm{Na}^{+}$ channel blockade by RS100642 has remarkable neuroprotective effects following global brain ischemia. In this context, RS100642 administration at a dose of $1 \mathrm{mg} / \mathrm{kg}$ following $5 \mathrm{~min}$ of global brain ischemia and 30 min of reperfusion significantly improves the MDA level and SOD and caspase-3 activities, all indicating free oxygen radical damage and apoptosis activation in the brain. However, further studies towards revealing the complete molecular mechanism underlying this neuroprotection, and determining the optimal ef- 
fective dose and time of administration, and potential benefits of co-administration of RS100642 with other drugs, remain to be conducted.

\section{Acknowledgments}

The authors would like to thank Roche Pharm. Co., USA for providing RS100642. This project was supported by a grant from the Scientific Research Coordination Department of Inonu University.

\section{Conflict of interest}

The authors declare no conflict of interest.

\section{References}

1. Sun J, Li T, Luan Q. Protective effects of delayed remote limb ischemic postconditioning: role for mitochondrial $\mathrm{K}_{\text {ATP }}$ channels in a rat model of focal cerebral ischemic reperfusion injury. J Cerebr Blood F Met 2012; 32: 851-9.

2. Distefano G, Pratico AD. Actualities on molecular pathogenesis and repairing processes of cerebral damage in perinatal hypoxic-ischemic encephalopathy. Ital J Pediatr 2010; 36: 63-72.

3. Lehotský J, Urban P, Pavlíková M. Molecular mechanisms leading to neuroprotection/ischemic tolerance: effect of preconditioning on the stress reaction of endoplasmic reticulum. Cell Mol Neurobiol 2009; 29: 917-25.

4. Woodruff TM, Thundyil J, Tang SC. Pathophysiology, treatment, and animal and cellular models of human ischemic stroke. Mol Neurodegener 2011; 6: 11-29.

5. Budihardjo I, Oliver H, Lutter M, Biochemical pathways of caspase activation during apoptosis. Cell Dev Biol 1999; 15: 269-90.

6. Linnik MD, Zobrist RH, Hatfield MD. Evidence supporting a role for programmed cell death in focal cerebral ischemia in rats. Stroke 1993; 24: 2002-8.

7. Jin R, Yang G, Li G. Inflammatory mechanisms in ischemic stroke: role of inflammatory cells. J Leukoc Biol 2010; 87: 779-89.

8. Ovbiagele B, Kidwell CS, Starkman S. Neuroprotective agents for the treatment of acute ischemic stroke. Curr Neurol Neurosci Rep 2003; 3: 9-20.

9. Williams AJ, Tortella FC. Neuroprotective effects of the sodium channel blocker RS100642 and attenuation of ischemia-induced brain seizures in the rat. Brain Res 2002; 932: 45-55.

10. Dave JR, Lin Y, Ved HS. RS-100642-198, a novel sodium channel blocker, provides differential neuroprotection against hypoxia/hypoglycemia, veratridine or glutamate-mediated neurotoxicity in primary cultures of rat cerebellar neurons. Neurotox Res 2001; 3: 381-95.

11. Williams AJ, Lu XCM, Hartings JA. Neuroprotection assessment by topographic electroencephalographic analysis: effects of a sodium channel blocker to reduce polymorphic delta activity following ischaemic brain injury in rats. Fundam Clin Pharmacol 2003; 17: 581-93.

12. Yao C, Williams AJ, Lu XCM. The sodium channel blocker RS100642 reverses down-regulation of the sodium channel alpha-subunit nav1.1 expression caused by transient ischemic brain injury in rats. Neurotox Res 2003; 5: 245-54.

13. Pulsinelli WA, Brierley JB. A new model of bilateral hemispheric ischemia in the unanesthetized rat. Stroke 1979; 10: 267-72.
14. Lowry OH, Rosebrough NJ, Farr AL, Randall RJ. Protein measurement with the folin phenol reagent. J Biol Chem 1951; 193: 265-75.

15. Mihara M, Uchiyama M. Determination of malonaldehyde precursor in tissues by thiobarbituric acid test. Anal Biochem 1978; 86: 271-8.

16. McCord JM, Fridovich I. Superoxide dismutase. An enzymic function for erythrocuprein. J Biol Chem 1969; 244: 6049-55.

17. Luck H. Catalase. In: Methods of Enzymatic Analysis. $2^{\text {nd }}$ edn. Bergmeyer HU (ed). Academic Press/Verlag Chemie, New York 1963; 885-94.

18. Lawrence RA, Burk RF. GSH-Px activity in rat liver. Biochem Biophys Res Commun 1976; 71: 952-8.

19. Grafe MR, Woodworth KN, Noppens K. Long-term histological outcome after post-hypoxic treatment with $100 \%$ or $40 \%$ oxygen in a model of perinatal hypoxic-ischemic brain injury. Int I Dev Neurosci 2008; 26: 119-24.

20. Radovsky A, Katz L, Ebmeyer U. Ischemic neurons in rat brains after 6,8 , or 10 minutes of transient hypoxic ischemia. Toxicol Pathol 1997; 25: 500-5.

21. Budwit-Novotny DA, McCarty KS, Cox EB. Immunohistochemical analyses of estrogen receptor in endometrial adenocarcinoma using a monoclonal antibody. Cancer Res 1986; 46: 5419-25.

22. Ottani A, Vergoni AV, Saltini S. Effect of late treatment with gamma hydroxybutyrate on the histological and behavioral consequences of transient brain ischemia in the rat. Eur J Pharmacol 2004; 485: 183-91.

23. Simao F, Matte A, Matte C. Resveratrol prevents oxidative stress and inhibition of Na+K+-ATPase activity induced by transient global cerebral ischemia in rats. J Nutr Biochem 2011; 22: 921-8.

24. Back T, Hemmen T, Schuler OG. Lesion evolution in cerebral ischemia. J Neurol 2004; 251: 388-97.

25. Candelario-Jalil E, Mhadu NH, Al-Dalain SM. Time course of oxidative damage in different brain regions following transient cerebral ischemia in gerbils. Neurosci Res 2001; 41: 233-41.

26. White BC, Sullivan JM, DeGracia DJ. Brain ischemia and reperfusion: molecular mechanisms of neuronal injury. J Neurol Sci 2000; 179 (Suppl 1-2): 1-33.

27. Kertmen H, Gürer B, Yilmaz ER. Antioxidant and antiapoptotic effects of darbepoetin-alpha against traumatic brain injury in rats. Arch Med Sci 2015; 11: 1119-28.

28. Björkman U, Ekholm R. Hydrogen peroxide degradation and glutathione peroxidase activity in cultures of thyroid cells. Mol Cell Endocrinol 1995; 111: 99-107.

29. Chelikani P, Fita I, Loewen PC. Diversity of structures and properties among catalases. Cell Mol Life Sci 2004; 61: 192-208.

30. Choi K, Kim J, Kim GW. Oxidative stress-induced necrotic cell death via mitochondira-dependent burst of reactive oxygen species. Curr Neurovasc Res 2009; 6: 213-22.

31. Clutton S. The importance of oxidative stress in apoptosis. Brit Med Bull 1997; 53: 662-8.

32. Lelli JL, Becks LL, Dabrowska MI. ATP converts necrosis to apoptosis in oxidant-injured endothelial cells. Free Radic Biol Med 1998; 25: 694-702.

33. Balla G, Vercellotti GM, Eaton JW. Iron loading of endothelial cell augments oxidant damage. I Lab Clin Med 1990; 116: 546-54.

34. Broughton BRS, Reutens DC, Sobey CG. Apoptotic mechanisms after cerebral ischemia. Stroke 2009; 40: 331-9.

35. Mehta SL, Manhas N, Raghubir R. Molecular targets in cerebral ischemia for developing novel therapeutics. Brain Res Rev 2007; 54: 34-66. 
36. Marton Z, Halmosi R, Horvath B. Scavenger effect of experimental and clinically used cardiovascular drugs. J Cardiovasc Pharmacol 2001; 38: 745-53.

37. Snider BJ, Gottron FJ, Choi DW. Apoptosis and necrosis in cerebrovascular disease. Oxidative/energy metabolism in neurodegenerative disorders. Ann NY Acad Sci 1999; 893: 243-53.

38. Cho S, Liu D, Gonzales C. Temporal assessment of caspase activation in experimental models of focal and global ischemia. Brain Res 2003; 982: 146-55.

39. Brahma MK, Dohare P, Varma S. The neuronal apoptotic death in global cerebral ischemia in gerbil: important role for sodium channel modulator. J Neurosci Res 2009; 87: 1400-11.

40. Namura S, Zhu J, Fink K. Activation and cleavage of caspase- 3 in apoptosis induced by experimental cerebral ischemia. J Neurosci 1998; 18: 3659-68.

41. Uzar E, Acar A, Evliyaoğlu O. The anti-oxidant and antiapoptotic effects of nebivolol and zofenopril in a model of cerebral ischemia/reperfusion in rats. Prog Neuropsychopharmacol Biol Psychiatry 2012; 36: 22-8.

42. Le DA, Wu Y, Huang Z. Caspase activation and neuroprotection in caspase-3 deficient mice after in vivo cerebral ischemia and in vitro oxygen glucose deprivation. Proc Natl Acad Sci 2002; 99: 15188-93.

43. Endres M, Namura S, Shimizu-Sasamata M. Attenuation of delayed neuronal death after mild focal ischemia in mice by inhibition of the caspase family. J Cereb Blood F Met 1998; 18: 238-47.

44. Qian L, Flood PM, Hong JS. Neuroinflammation is a key player in Parkinson's disease and a prime target for therapy. J Neural Transm 2010; 117: 971-9.

45. Nguyen TA, Frank-Cannon TC, Martinez TN, et al. Analysis of inflammation-related nigral degeneration and locomotor function in DJ-1-/- mice. J Neuroinflammation 2013; 10: 50-2. 